\title{
Testicular Metastasis as an Initial Manifestation of Gastrointestinal Cancer
}

\section{Soumia Berrad ${ }^{*}$, Zineb Benbrahim ${ }^{1}$, Vincent Lorenga Kazadi', Lamiae Nouiakh ${ }^{1}$, Hayat Erraichi' ${ }^{1}$, Rafik Naas ${ }^{2}$, Lamiae Amaadour ${ }^{1}$, Karima Oualla ${ }^{1}$, Samia Arifi ${ }^{1}$, Hinde El Fatemi ${ }^{2}$, Nawfel Mellas ${ }^{1}$}

${ }^{1}$ Department of Oncology Medical, Hassan II University Hospital, Fès, Morocco

${ }^{2}$ Department of Anathomopathology, Hassan II University Hospital, Fès, Morocco

Email: *soumiaberrad3@gmail.com

How to cite this paper: Berrad, S., Benbrahim, Z., Kazadi, V.L., Nouiakh, L., Erraichi, H., Naas, R., Amaadour, L., Oualla, K., Arifi, S., El Fatemi, H. and Mellas, N. (2021) Testicular Metastasis as an Initial Manifestation of Gastrointestinal Cancer. Journal of Cancer Therapy, 12, 57-64. https://doi.org/10.4236/jct.2021.121006

Received: December 4, 2020

Accepted: January 18, 2021

Published: January 21, 2021

Copyright $\odot 2021$ by author(s) and Scientific Research Publishing Inc. This work is licensed under the Creative Commons Attribution International License (CC BY 4.0).

http://creativecommons.org/licenses/by/4.0/

\begin{abstract}
Metastatic small bowel tumors in the testes are uncommon often indicating an advanced stage of sickness with poor prognosis. The route of spread to the scrotal level has not yet been defined. The most of these tumors are diagnosed at autopsy or orchiectomy for metastatic carcinoma. The diagnosis is mainly based on histological and immunohistochemical data. In this paper, we report the case of a 36-year-old patient with a spermatic torsion secondary to a testis metastasis from an adenocarcinoma of the small intestine confirmed histologically, occurring as first clinical manifestation that was taken care in the medical oncology department of CHU Hassan II in Fès. The aim of the subject is to highlight this rare metastatic localization of gastrointestinal cancer; how to approach the diagnosis and distinguish between primary testicular cancer and testicular metastasis; by describing the different clinical, radiological and anatomopathological aspects of metastatic gastrointestinal cancer to the testis.
\end{abstract}

\section{Keywords}

Adenocarcinoma, Hail Intestine, Metastasis, Testes, Chemotherapy

\section{Introduction}

Cancers of the hail intestine remain rare and represent less than $5 \%$ of digestive cancers [1], although the small intestine represents $75 \%$ of the length of the digestive tract and $90 \%$ of its mucous surface. The incidence of these tumors has been increasing in recent decades; the diagnosis is often made at an advanced 
stage due to nonspecific symptomatology.

Gastrointestinal cancers usually spread to the liver, lungs and bones. Testicular metastases from a primary digestive system are uncommon; very few cases have been reported; Metastatic testicular tumors accounting for approximately $2 \%$ of all testicular neoplasms [2]. The fact that it is progressive and clinically indicative of gastrointestinal cancer is also an unusual circumstance. The route of spread to the scrotal level has not yet been defined, the lymphatic and retrograde peritoneal route are involved according to some authors.

The diagnosis is mainly based on histological and immunohistochemical data from a biopsy or orchidectomy. Because testicular metastasis of the hail intestine (TMHI) is rare, also the few cases revealed in the literature are not sufficient to define the recommendations and therapeutic strategies. Treatment for TMHI is usually dependent on the stage of the disease. The therapy often involved different modalities such as surgery, chemotherapy and radiation therapy. The prognosis also depends on the stage but it remains unfavorable.

We report this case to highlight the rarity of this secondary localization of small bowel carcinoma, the clinico-radiological and pathological characteristics, in particular the misleading presentation mimicking a primary neoplasm of the testis, the management of the treatment and the prognosis.

\section{Observation}

This is a 36-year-old patient, with no particular pathological history or gastrointestinal symptoms, who presented one month before his admission with acute onset right hemispherical pain without associated urinary signs, the patient consulted urgently. Physical examination objectified a hard firm right scrotal mass painful on palpation simulating a torsion of the spermatic cord. The scrotal ultrasound showed a centimetric tissular mass. Alpha-fetoprotein and beta-chorionic gonadotropin (HCG) dosages were within normal limits.

Surgical exploration was done and completed with biopsies from the right scrotal mass.

The histopathological examination concluded that it is a scrotal localization of an adenocarcinoma (Figure 1).

An immunohistochemical study was performed, the tumor cells express the anti-CKAE1/AE3 antibody and weakly and focally the anti-CK20 and anti-CDx2 antibodies. They do not express anti-CK7, anti-Chromogranin, anti-Synaptophysin or anti-Calretinin antibodies (Figure 2).

The immunohistochemical profile confirmed the gastrointestinal origin.

The patient underwent an extension assessment made by the thoraco-abdomino-pelvic scanner which showed a tumor-like tissue process of the last ileal loop, of the ileo-colic junction responsible for a small occlusion with ileo-colic lymphadenopathy (Figure 3) and a right scrotal tissue nodule enhanced after contrast compatible with a malignant lesion (Figure 4) and secondary hepatic and pulmonary and peritoneal localization. 


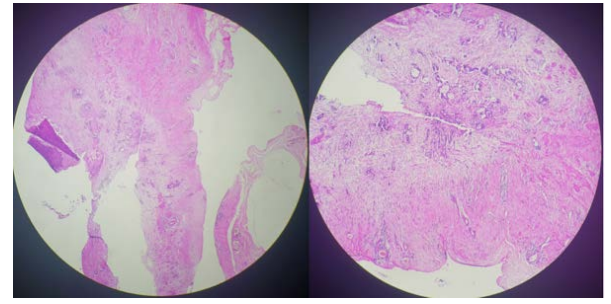

(a)

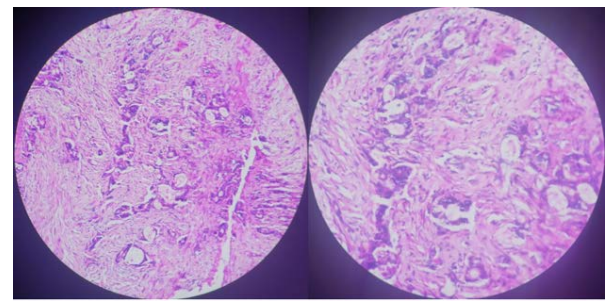

(b)

Figure 1. (a) Histologycall image showing Tumor proliferation of glandular architecture made up of small cells, variable in shape with eosinophilic cytoplasm and a small, hyperchromatic nucleus; (b) Histologycall image showing Presence of some figures of mitosis and the tumor stroma is predominantly fibrous.

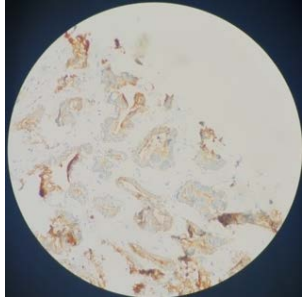

(a)

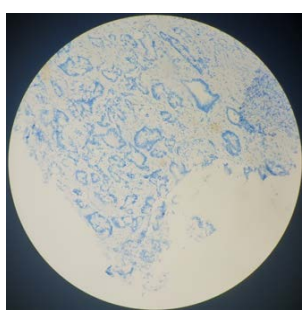

(c)

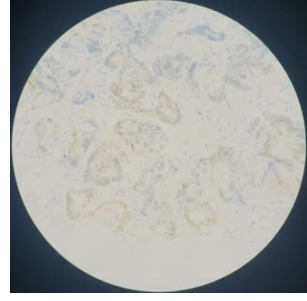

(b)

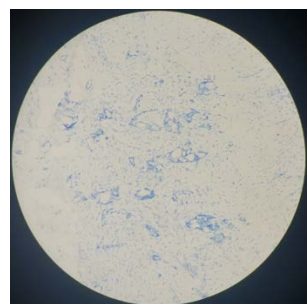

(d)

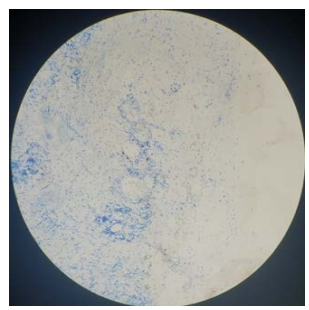

(e)

Figure 2. (a) Immunohistochemestry showing intensive positive expression of CK AE1/AE3; (b) Immunohistochemestry revealing Low intensity nuclear labeling of tumor cells for CDX 2; (c) Immunohistochemestry revealing negative staining for Calritinin; (d) Immunohistochemestry revealing negative staining for Synaptophysin; (e) Immunohistochemestry showing negative staining for Chromogranin. 


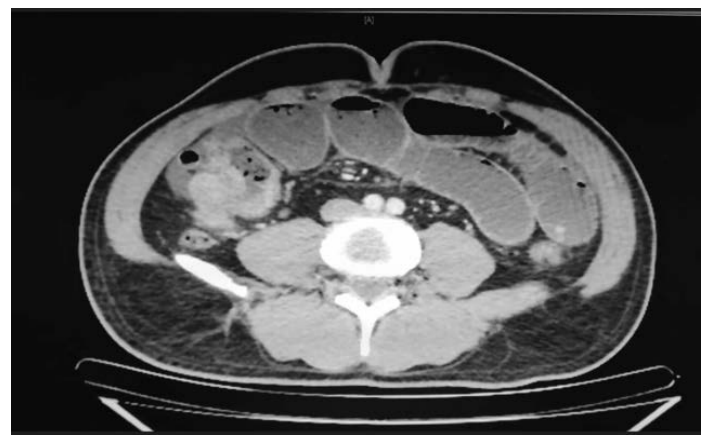

Figure 3. Abdominal CT scan showing digestive thickening of the last ileal loop, irregular circumferential responsible for stenosis of the digestive lumen. with abdominal lymphadenopathy with multiple peritoneal tissue nodules.

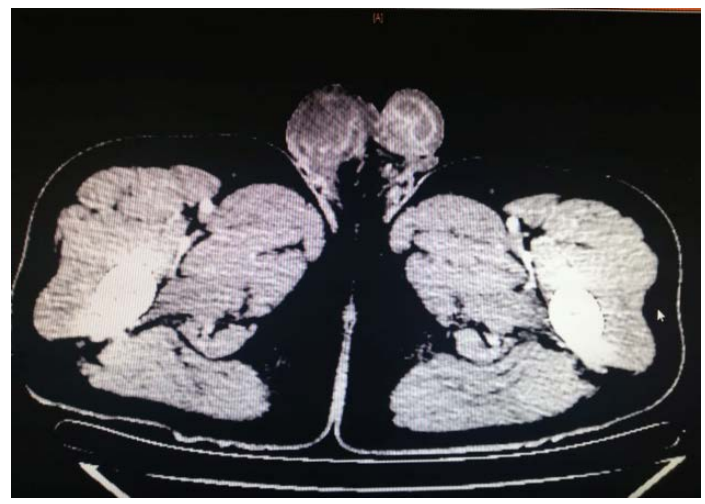

Figure 4. Pelvic CT scan revealing a right scrotal tissue nodule enhanced after contrast compatible with a malignant lesion.

The digestive tumor markers were strongly positive CA19-9 at $430 \mathrm{U} / \mathrm{ml}$ and ACE $18.9 \mathrm{U} / \mathrm{ml}$ after two weeks of the surgical procedure, the patient presented with intestinal obstruction put under rehydration with corticosteroid therapy with good progress.

Considering adenocarcinoma of hail intestine as a primary focus, a palliative treatment was initiated with chemotherapy containing 5FU, oxaliplatin after three cures he presented a clinical improvement with radiological stability, then the evolution was marked by rapid deterioration of patient's intestinal with a perforation with peritonitis admitted to the operating room where he presented hemodynamic instability leading to the death of patient.

\section{Discussion}

Adenocarcinomas of the small intestine are rare, accounting for less than $2 \%$ of digestive tumors [1]. The diagnosis is often made at an advanced stage due to not very specific symptoms [2], which makes the prognosis of these entities also poor, with a 5-year survival of less than $30 \%$ and a median survival of 19 months [3].

Adenocarcinoma of the small intestine is a multifactorial pathology, the risk factors that have been identified in epidemiological studies for developing a tu- 
mor of the intestine are the following, genetic predispositions in particular the PAF linked to a mutation of the APC gene characterized by the appearance of multiple adenomatous Duodenal polyps that degenerate into adenocarcinoma, inflammatory bowel disease, excessive consumption of alcohol and tobacco [4] [5].

The most common symptom at the time of diagnosis is abdominal pain $67 \%$ [5].

However, the diagnosis can be made in an emergency setting such as intestinal obstruction (40\%) or digestive bleeding [6].

Approximately one third of patients present with distant metastases (stage IV) and one third with lymph node involvement (stage III) at the time of diagnosis [7].

Testicular metastasis from cancer of the digestive tract is a rare or even exceptional eventuality, as conventionally three organs can be affected, especially the omental lymph node chains, the peritoneum and the liver [8].

The incidence of testicular metastases is estimated at $2.5 \%$ according to various series of autopsies. With the exception of infiltration of leukemia and lymphoma, secondary neoplasms of the testes are unusual, with an incidence of $0.02 \%-2.5 \%[8]$.

Clinical cases reported in the literature analyzed the testicular metastases from gatrointestinal cancer showing The age of the patients ranged from 18 to 76 years (mean 51), with average survival is 6 - 12 months after the diagnosis [5] [9]. However, at the stage of testicular metastases there is often simultaneous tumor dissemination to other organs, and exceptionally the testicular tumor was the first manifestation of the disease [9].

The most of these tumors are diagnosed at autopsy or orchiectomy. Their evident clinical manifestations upon clinical can guide discovery of the primary tumor and histopathological examination of testicular tissue may lead to the identification of carcinoma of digestive or other origin [9].

Another study analyzed at 127 reports of heterogeneous testicular metastasis and concluded that the cancers metastasized to the testis are prostate cancer (35\%), lung cancer (19\%), malignant melanoma (9\%), gastrointestinal cancer (8.6\%) and renal carcinoma (7.8\%). Testicular metastases were bilateral in $15 \%$ of cases [5] [10] of these only 2 cases reported adenocarcinoma of the small intestine metastatic to the testes.

Although the mechanism of testicular metastasis remains unknown, there are several routes by which primary gastrointestinal carcinomas metastasize to the testes. direct invasion of adjacent lesions, retrograde venous embolism, arterial embolization, retrograde lymphatic extension from para-aortic lymph nodes, transperitoneal seeding through a congenital hydrocele or retrograde extending from the vas deferens [11] [12], Many cases in the reported literature suggest the concept of retrograde lymphatic spread [12] [13]. However, another author has also suggested the spread through the spermatic veins due to extension of the 
tumor via the retro peritoneum [14].

The immunohistochemical profile should show a positivity of CDX2 cytokeratin 20, the negativity of cytokeratin 7 to confirm the diagnosis of testicular metastasis of digestive origin [14]. In our case, the diagnosis was also based on the previous markers. Since TMHIs are unusual, there are no randomized clinical trials to determine the optimal treatment for localized stages; The most common treatment modalities in the various cases reported were radical primary and metastasis surgery combined with adjuvant chemotherapy.

In metastatic cases, systemic chemotherapy is essential. Few protocols have been evaluated by mainly retrospective studies including $5 \mathrm{FU}$. The combination of 5FU and platinum salts seem to provide an improvement in overall survival [15].

The largest retrospective multicenter study conducted by A zaanan included ninety-tree patients with small-bowel adenocarcinoma advanced compared chemotherapy with $5 \mathrm{FU}$ alone to the combination of $5 \mathrm{FU}$ with platinum salt suggests a significant improvement in overall survival in the arm platinum-based chemotherapy regimen with 17.0 v 12.7 months [16]. Recently a French multicenter retrospective series included 95 patients treated with FOLFOX, LV5FU, LV5FU-cisplatin or FOLFIRI. The median survival was 15.1 months.

Patients treated with first-line FOLFOX had the best survival (17.8 months) [17].

Finally a phase II trial reported encouraging results of the combination oxaliplatin + capecitabine in 30 patients with $52 \%$ objective responses and the median overall survival was 20.4 months. Capeox should be considered a standard regimen for advanced small bowel [18]. In the NADEGE cohort, the 1st line chemotherapy was FOLFOX in $80 \%$ of cases, FOLFIRI in $12 \%$ and LV5FU2 in $5 \%$ [19]. In the event of peritoneal carcinoma Cytoreductive surgery combined with intraperitoneal chemotherapy gives satisfactory survival results. When possible, this combined approach should become the standard treatment. This cumbersome and not yet standardized procedure is only intended for selected patients in good general condition, in whom the carcinoma is macroscopically resectable, reserved for expert centers [20].

Despite therapeutic progress, the prognosis remains poor, especially in the presence of generalized peritoneal disease.

\section{Conclusion}

Testicular metastases from adenocarcinoma of the hail intestine are unusual where light microscopy and immunohistochemistry helped to reach the correct diagnosis; with a pathogenesis that is still unclear, it often signals an advanced stage of the disease with an unfavorable prognosis. The differential diagnosis of testicular cancer should be considered in a patient who presents with testicular symptoms an adequate therapeutic strategy must be proposed especially in the early stages. 


\section{Conflicts of Interest}

The authors declare no conflicts of interest regarding the publication of this paper.

\section{References}

[1] Neugut, A.I., Jacobson, J.S., Suh, S., Mukherjee, R. and Arber, N. (1998) The Epidemiology of Cancer of the Small Bowel. Cancer Epidemiology, Biomarkers \& Prevention, 7, 243-251.

[2] Aparicio, T., Zaanan, A., Svrcek, M., Laurent-Puig, P., Carrere, N., Manfredi, S., Locher, C. and Afchain, P. (2013) Small Bowel Adenocarcinoma: Epidemiology, Risk Factors, Diagnosis and Treatment. Digestive and Liver Disease, 46, 97-104. https://doi.org/10.1016/j.dld.2013.04.013

[3] Howe, J.R., Karnell, L.H., Menck, H.R. and Scott-Conner, C. (1999) The American College of Surgeons Commission on Cancer and the American Cancer Society. Adenocarcinoma of the Small Bowel: Review of the National Cancer Data Base, 1985-1995. Cancer, 86, 2693-2706. https://doi.org/10.1002/(SICI)1097-0142(19991215)86:12<2693::AID-CNCR14>3.0. $\underline{\mathrm{CO} ; 2-\mathrm{U}}$

[4] Jess, T., Loftus Jr., E.V., Velayos, F.S., Harmsen, W.S., Zinsmeister, A.R., Smyrk, T.C., et al. (2006) Risk of Intestinal Cancer in Inflammatory Bowel Disease: A Population-Based Study from Olmsted County Minnesota. Gastroenterology, 130, 10391046. https://doi.org/10.1053/j.gastro.2005.12.037

[5] Verma, N., Babu, S., Kushwaha, J.K. and Singhai, A. (2013) Testicular Metastasis of Colorectal Carcinoma: An Unusual Presentation. BMJ Case Reports, 2013.

[6] Bulow, S., Alm, T., Fausa, O., Hultcrantz, R., Jarvinen, H., Vasen, H., et al. (1995) Duodenal Adenomatosis in Familial Adenomatous Polyposis. International Journal of Colorectal Disease, 10, 43-46. https://doi.org/10.1007/BF00337586

[7] Cabanne, F. and Bonenfant, J.L. (1986) Pathological Anatomy: Principles of General Pathology, Special Pathology and Aetopathology. Maloine SA Publisher, Paris.

[8] Kusaka, A., Koie, T., Yamamoto, H., Hamano, I., Yoneyama, T., Hashimoto, Y., et al. (2014) Métastases testiculaires du cancer de la prostate: Un rapport de cas. Rapports de cas en Oncologie, 7, 643-647. https://doi.org/10.1159/000367779

[9] Tozawa, K., Akita, H., Kusada, S. and Yutaro, H. (2015) Testicular Metastases from Carcinoma of the Bile Duct: A Case Report. World Journal of Gastroenterology, 21, 6764-6768.

[10] Grignon, D.J., Shum, D.T. and Hayman, W.P. (1986) Metastatic Tumours of the Testes. Canadian Journal of Surgery, 29, 359-361.

[11] Dabaja, B.S., Suki, D., Pro, B., Bonnen, M. and Ajani, J. (2004) Adenocarcinoma of the Small Bowel: Presentation, Prognostic Factors, and Outcome of 217 Patients. Cancer, 101, 518-526. https://doi.org/10.1002/cncr.20404

[12] Thompson, G.J. and Pilcher, F. (1935) Carcinoma of the Vas Deferens: Report of a Case. Journal of Urology, 34, 714-717. https://doi.org/10.1016/S0022-5347(17)72323-3

[13] Ulbright, T.M. and Young, R.H. (2008) Metastatic Carcinoma to the Testis. A Clinicopathologic Analysis of 26 Nonincidental Cases with Emphasis on Deceptive Features. The American Journal of Surgical Pathology, 32, 1683-1693.

[14] Meacham, R.B., Mata, J.A., Espada, R., Wheeler, T.M., Schum, C.W. and Scardino, 
P.T. (1988) Testicular Metastasis as the First Manifestation of Colon Carcinoma. Journal of Urology, 140, 621-622. https://doi.org/10.1016/S0022-5347(17)41740-X

[15] Locher, C., Malka, D., Boige, V., Lebray, P., Elias, D., Lasser, P. and Ducreux, M. (2005) Combination Chemotherapy in Advanced Small Bowel Adenocarcinoma. Oncology, 69, 290-294. https://doi.org/10.1159/000089678

[16] Zaanan, A., Costes, L., Gauthier, M., Malka, D., Locher, C., Mitry, E., et al. (2010) Chemotherapy of Advanced Small Bowel Adenocarcinoma: A Multicenter AGEO Study. Annals of Oncology, 21, 1786-1793. https://doi.org/10.1093/annonc/mdq038

[17] Overman, M.J., Kopetz, S., Wen, S., Hoff, P.M., Fogelman, D., Morris, J., Abbruzzese, J.L., Ajani, J.A. and Wolff, R.A. (2008) Chemotherapy with 5-Fluorouracil and a Platinum Compound Improves Outcomes in Metastatic Small Bowe Adenocarcinoma. Cancer, 113, 2038-2045. https://doi.org/10.1002/cncr.23822

[18] Overman, M.J., Varadhachary, G.R., Kopetz, S., Adinin, R., Lin, E., Morris, J.S., et al. (2009) Phase II Study of Capecitabine and Oxaliplatin for Advanced Adenocarcinoma of the Small Bowel and Ampulla of Vater. Journal of Clinical Oncology, 27, 2598-2603. https://doi.org/10.1200/JCO.2008.19.7145

[19] Aparicio, T., et al. (2013) NADEGE Prospective Cohort Demographic Data of 335 Patients with Small Bowel Adenocarcinomas. European Cancer Congress, A2466.

[20] Elias, D., Glehen, O., Pocard, M., Quenet, F., Goéré, D., Arvieux, C., Rat, P. and Gilly, F. (2010) Association Française de Chirurgie. 